\title{
Near Real Time Analytic Processing of Traffic Data Streams
}

\author{
Paulo Pintor \\ paulopintor@ua.pt \\ DETI, University of Aveiro \\ Portugal
}

\author{
Rogério Luís de C. Costa \\ rogerio.l.costa@ipleiria.pt \\ CIIC, Polytechnic of Leiria \\ IEETA, University of Aveiro \\ Portugal
}

\author{
José Moreira \\ jose.moreira@ua.pt \\ DETI - IEETA, University of Aveiro \\ Portugal
}

\begin{abstract}
Location data is vital for traffic management and for transportation and urban planning, but also benefits people in daily life, helping on decisions related to route planing and on the use of public transportation. Although historical data can provide insights on expected traffic volume at a certain region and time, predictions based solely on historical data fail to deal with events like street works and traffic-accidents. In this work, we use real time information together with historical data to predict traffic by road segment in the near future. The paper outlines the architecture of the system, the data model and the prediction method. Preliminary results using real world data on taxi positions show that using stochastic processes is a promising approach for short-term traffic forecasting.
\end{abstract}

\section{CCS CONCEPTS}

- Information systems $\rightarrow$ Mobile information processing systems; Data streaming; Location based services.

\section{KEYWORDS}

Traffic prediction, GPS data, Data Streams.

\section{ACM Reference Format:}

Paulo Pintor, Rogério Luís de C. Costa, and José Moreira. 2020. Near Real Time Analytic Processing of Traffic Data Streams. In 13th International Workshop on Computational Transportation Science (IWCTS'20), November 3, 2020, Seattle, WA, USA. ACM, New York, NY, USA, 4 pages. https://doi.org/ $10.1145 / 3423457.3429365$

\section{INTRODUCTION}

Traffic estimation is a key feature in urban and public transportation planning. Tools to collect traffic data include loop sensors, surveillance cameras, and floating cars, i.e., vehicles equipped with Global Positioning System (GPS) receivers. The use of floating car based traffic monitoring has emerged some years ago as a promising approach, especially because a wide range of day-to-day technologies incorporated location information and several cities started to collect GPS data from public transportation, like taxicabs and buses. Taxi drivers are considered experienced drivers who can find quick routes based on their knowledge [13] and, thus, taxicab GPS datasets have been used in several contexts, including route

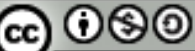

This work is licensed under a Creative Commons Attribution-NonCommercial-ShareAlike International 4.0 License.

IWCTS'20, November 3, 2020, Seattle, WA, USA

(C) 2020 Copyright held by the owner/author(s).

ACM ISBN 978-1-4503-8166-6/20/11.

https://doi.org/10.1145/3423457.3429365 planning [12], to detect unexpected behaviors in traffic flow [7] and to anomalous events (e.g., accidents) [14].

But using such historical data to predict traffic volume is not a trivial task. Several factors may impact on traffic volume, including the spatial context (e.g. the presence of points of interest), the temporal context (e.g. holidays and rush hours), the weather and road condition. Also, traffic volume prediction based solely on historical data fail to deal with variations related to common occurrences like road works, accidents, traffic lights that don't work properly, sudden changes in weather, and so on.

In this work, we propose a traffic prediction system that uses widely available GPS data to learn traffic patterns and to keep statistical data on road traffic always up to date. We use taxicabs GPS historical data to build a prediction model and near real time GPS data to adjust predictions to to the current status of traffic. Our model is capable to predict the number of taxicabs by road segment in the near future. The main contributions include (i) a traffic prediction model, (ii) its use on a system capable to process traffic related data streams in near real time and (iii) an actual validation on real world data.

The next section presents an overview of the related work. Section 3 describes the proposed traffic prediction model and the data stream processing system. Section 4 presents experimental results obtained using real world data. Finally, section 5 concludes the paper and describes future work.

\section{RELATED WORK}

Traffic prediction can be used to improve drivers satisfaction, to propose routes, in urban and public transportation planning, and to identify anomalous events or behaviors (e.g. traffic collision and unexpected stops). As real-world traffic data can be obtained from distinct sources (like loop sensors, vehicles equipped GPS receivers and even cell phones), many works use pattern recognition on such data to make future predictions. In [8], authors estimate future traffic flow using data on the speed of probe vehicles (gathered from navigation devices at certain measure points) together with exact data about traffic collected using loop sensors. They compare the traffic estimated using the data from the probe vehicles with the exact traffic gathered from traffic-loop detectors to identify how representative are the sample data and use this information to estimate the traffic in locations where there is no loop detectors. There is no explanation on how to estimate sample data representativeness in road segments where there are no loop detectors and on how sudden events (e.g. collisions) affect historical rates.

Loop sensors can be used to collect traffic data, but they are expensive and mostly available in major highways and roads. They are not used much in cities [6,13]. On the other hand, GPS-equipped taxicabs have been generating large amounts of data on traffic flow. 
Meng et al [6] use loop detector data together with GPS data from taxicabs to estimate traffic volume. They map GPS trajectories onto a road network, infer the average travel speed and time at each segment, and use learned speed patterns to build a volume affinity graph. This is used together with loop detector data to train supervised and semi-supervised methods (e.g. linear regression, random forest and a multi-layer perceptron network). There is no mention on how to deal with the current state of the traffic.

In $[11,12]$, Yuan et al. present the T-Drive system. In the first version of T-Drive, historical data on taxicab trajectories is used to choose which is fastest route to a certain destination at a given departure time. Authors show that using such historical data may be more effective than using shortest-path and real-time-trafficbased methods, but agree that the of historical data alone would fail, for instance, in situations that the system is used for many users to choose for a certain route in the same time window. In such situations, the same route would be recommended for all users even though a road become crowded. Then, in [10], the authors propose a routing system by using recent traffic information together with historical data to estimate travel times, to propose driving directions customized for a particular user. The proposed model also incorporates weather condition information, but the focus is on learning the drivers' behavior from historical data. The paper also does not address the problem of processing the data flow in an efficient and scalable way. Other works on travel time estimation and route recommendation include [4, 9].

Pang et al. [7] use taxicab GPS data to monitor the existence of unexpected behavior in traffic flows. They consider that cities are partitioned as a grid and use statistical methods to foreseen the number of vehicles in each grid cell. Then, GPS data on taxis position is used to identify anomalous number of vehicles in each grid cell. Their work focus on traffic outliers detection in historical data. Outlier detection in traffic data is also studied in $[2,5]$

In [3], mobile phone data is used together with loop detectors to estimate traffic volume. Authors use regression models and state that using networks handover can provide more accurate estimations of volume in a specific site where there is no traffic data information than relying on average city-wide traffic volume. A limitation of their proposal is that the handover event only occurs at phones that are actually making calls and that calls' duration must be long enough to traverse the boundaries of two network cells. Other works $[1,3]$ also use cell phone data to estimate traffic related information, like traffic status and vehicle speed.

In our work, we use GPS traces to build an historical database used to identify traffic patterns but also to support the detection of near real time variations on predicted traffic pattern. This way, our model can deal with periodic patterns and sudden events, like collisions and unexpected stops.

\section{TRAFFIC PREDICTION PROPOSAL}

This section presents the architecture and the method proposed to predict the number of taxicabs in a road segment.

\subsection{Architecture}

We use real time GPS data gathered from moving vehicles (e.g. taxicabs) to compile a historical database and to support predictions.
These data are sent to a stream processing system to prepare the location data and the results are forwarded to a Web Service. Whenever a user (e.g., a driver or a traffic management authority) wishes to know the traffic volume in the near future at a specific road, it is enough to send a request to the Web Service.

Stream processing system - The data processing system is implemented in Apache Storm, an open source, distributed and horizontally scalable real-time computation system. The key elements in Storm are Spouts and Bolts. The role of Storm in our architecture is to receive the data through the Spouts and send the traffic data to the Bolts. Bolts perform the pre-processing tasks and they also prepare the data about the last known position (road segment) of a vehicle. The Web Service uses that information to create counters and other data needed for prediction and visualization.

Web Service - The Web Service manages several data structures holding all data about the current state of the traffic and other data needed to implement the predictive algorithm.

\subsection{Concepts and data model}

The source data are GPS observations tracking the location of vehicles along time (historical data) and a transportation network. The latter is a directed graph $G=(V, E)$, where the vertices (nodes) $V$ are road intersections (hereafter denoted intersections) and the edges $E$ represent the path between two intersections (hereafter denoted segments), with $E \subseteq V \times V$. The set of observations is $O=(C, T, P)$, such that $C$ is the set of vehicles, $T$ is the set of timestamps and $P$ is the set of GPS locations. So, we need a map-matching function $F(P) \rightarrow E$ to assign each GPS location $p \in P$ to a segment $e \in E$ of the transportation network $G$. The result is $\bar{O}=(C, T, E)$. The connectivity of the segments in $G$ is a square matrix $M t$, where the rows and columns are the segments in $E$ and the values are "1" or "0", meaning, respectively, if two segments are connected by an intersection or not. This model assumes that a vehicle can only move from a segment to another segment through an intersection and events like U-turn are ignored.

We build a transition matrix Mp for each intersection $(v \in V)$. The rows and columns are the segments connected to $v$, and the values stand for the probability of a vehicle moving from segment $e_{i}$ to segment $e_{j}$ at $v$. This requires a step to scan all tuples in $\bar{O}$ and create a matrix Mc counting the number of vehicles moving from a segment to another segment for each intersection and a step to scan the matrices Mc of each intersection and compute the corresponding matrices $\mathrm{Mp}$ with the transition probabilities (1).

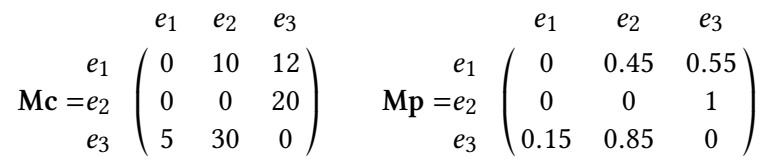

The probabilities in Mp are percentages obtained from Mc row by row using (2), such that $n$ stands for the number of segments connected to $v$.

$$
P_{l, c}=\frac{C_{l, c}}{\sum_{j=l}^{n} x_{l, j}}
$$

For instance, the probability for $l=1$ and $c=2$, i.e., the probability of a vehicle in $e_{1}$ turning to $e_{2}$ is $\approx 0.45$. 
The transition matrices allow to analyze the traffic flow in road segments and intersections. They are created offline using the GPS locations and they can be updated periodically.

\subsection{Prediction method}

The model defined above is prepared to implement several methods of traffic forecasting. The focus here is to predict the number of vehicles that will be in a segment in the near future using offline and real-time data. For this purpose, GPS observations $\bar{O}$ are split into two subsets. Assuming that the observations are ordered by timestamp, the first $k$ observations are used to construct the transition matrices by intersection (offline data), as defined in 3.2, and the remaining $m$ observations are used to simulate the state (number of vehicles per segment) of a transportation network in real time.

Real-time data consists of two collections (dictionaries): $L e=$ $\{E:<C>\}$ where the key is a segment $e \in E$ and the value is a set of vehicles $\langle c\rangle$ (with $c \in C$ ) moving in that segment at a given time, and $L c=\{C: E\}$ where the key is a vehicle $c \in C$ and the value is the last segment where that vehicle was observed for the last time. As all collections are initially empty, we need a warm-up phase to fill the collections $L e$ and $L c$ and obtain a realistic state of the traffic in the road network. The observations in $\bar{O}$ are traversed starting at position $m$, and for every $\bar{o}=(c, t, e)$ the following steps are performed: 1) the last position $e^{\prime}$ of the vehicle $c$ is retrieved from $L c$, the entry $c$ is removed from the set of vehicles moving in $e^{\prime}$ of $L e$ and a new entry $c$ is added to the set of vehicles moving in $e$ of $L e$; and 2) the entry ( $c: e$ ) of $L c$ is updated. At the end of the warm-up procedure, it is possible to know how many vehicles are in each segment at a given time.

The number of vehicles in a given segment in a near future is estimated using a recursive algorithm. The parameters are a segment (target) and the number of recursive iterations (depth). This also defines the sub-graph of $G$ to be processed. In the first iteration, the recursive method retrieves only the segments that are directly connected to the target. The second iteration repeats the process for all segments retrieved in the previous step, and so on. At each step, the transition matrices of each intersection are used to estimate the number of vehicles that enter and leave each segment of the sub-graph (Figure 1).

The algorithm must avoid cycles and so, the result is a tree of segments with a predefined depth. As the computationally complexity of the algorithm and the errors of the estimation increase with depth, the number of recursive iterations should be small.

\section{EXPERIMENTAL EVALUATION}

In order to evaluate our proposal, we performed several tests using a dataset with GPS information of taxicabs in the the city of Beijing (China) $[10,12]$. This dataset has $3 \mathrm{~GB}$ and the attributes are: (i) taxicab identification, (ii) taxicab position (lat, lon), (iii) timestamp and (iv) true if the taxicab had passengers inside. The subset we use has 578,474 records of 551 distinct taxicabs in 10,098 different segments in a specific region of the city. Our database also contains information about segments and intersections resembling a map of the city. The original data was obtained from OpenStreet Maps. We perform a map matching task to assign each taxicab position to a road segment in the Beijing map, and split the dataset into training and validation sets.

To simulate the GPS devices sending data about their location, we built a module emulating the cars riding on the roads and sending this data to our stream processing system. We built a SOAP web service and a web application to consume the service. The application is built in JAVA, JAVA EE and HTML5 and allows creating trajectories and providing information on traffic flow using color schemes. PostgreSQL is used to store the database.

\subsection{Trajectory Suggestion and Traffic Flow Representation}

In our web application, users can find roads or look for trajectory suggestions (created by the system) using a map or by specifying addresses. Specified roads or trajectory suggestions are displayed on a map. Users may also request additional information, like the number of taxis in a road segment during the last hour or an estimation of a near real time situation.

The system uses colors to represent segments depending on the comparison between near real time and historical data. Let $\rho$ be the average number of vehicles in a segment computed using historical data and $\gamma$ be the vehicle count in such segment in nearly real time. When $\rho>=\gamma$ the segment is coloured green. If $\rho<\gamma<(\rho * 1.25)$ then the segment is coloured yellow. Otherwise, the segment is coloured red. In the example of Figure 2, the user selected a single segment (coloured red). As the user selected just one segment, the system coloured the selected segment but also its adjacent segments (which may provide useful information to the user).

\subsection{Traffic Estimation}

The estimation of the number of taxis by road segment was evaluated in two regions of Beijing. We converted the OSM maps into a graph using the OSM to routeware application. By data profiling, we found that a taxi takes approximately 7 minutes to traverse 3 segments, and so, the example presented in this work, is to estimate the number of taxis that will be in a road segment within 7 minutes.

The results are displayed in Figure 3. These estimated values are always greater than the actual number of taxis in a road segment with an average difference of $13,5 \%$ and a standard deviation of $1.2 \%$. However, as the elapsed time between consecutive observations of the same taxicab is sometimes greater than 10 minutes, the taxicabs could have been in several segments that were not reported in the system, and so the results can be slightly better than presented.

\section{CONCLUSIONS AND FUTURE WORK}

Traffic volume estimation is important in several contexts, including in urban and public transportation planning. Although historical data can be used to identify some relevant patterns, near real time observations are important to take into consideration the most recent status of a road network.

In this paper, we present a scalable system that uses GPS data to build a historical database and also as near real time information for traffic volume prediction. The use case shows how to use the system to predict traffic volume in near future, but the data model and prepossessing tasks can also be used to build other short-term 


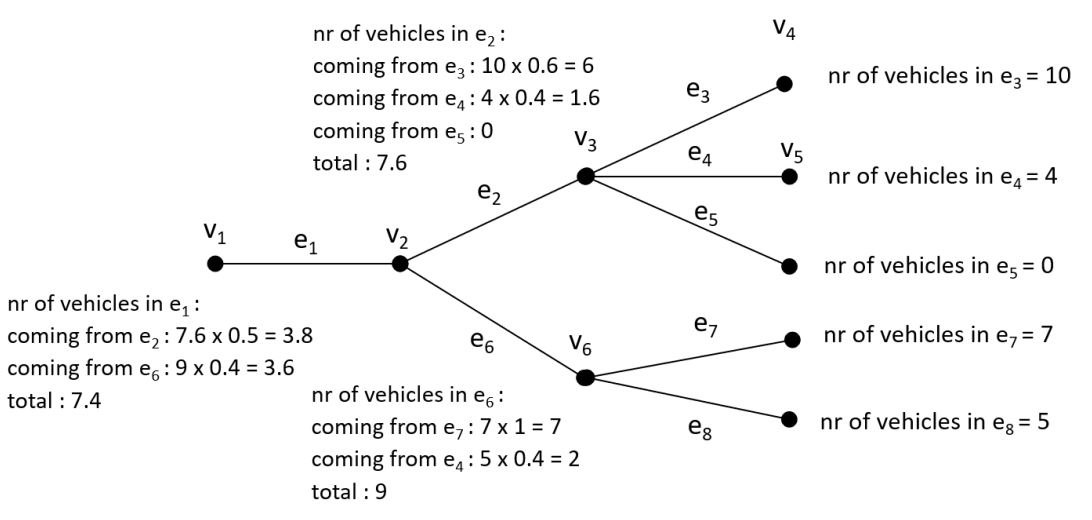

\begin{tabular}{|c|c|c|c|c|}
\hline \multicolumn{5}{|c|}{ intersection $v_{2}$ probability matrix } \\
\hline & $e_{1}$ & $\mathrm{e}_{2}$ & \multicolumn{2}{|c|}{$e_{6}$} \\
\hline$e_{1}$ & 0 & 0.3 & \multicolumn{2}{|l|}{0.7} \\
\hline$e_{2}$ & 0.5 & 0 & \multicolumn{2}{|l|}{0.5} \\
\hline$e_{6}$ & 0.4 & 0.6 & \multicolumn{2}{|l|}{0} \\
\hline \multicolumn{5}{|c|}{ intersection $v_{3}$ probability matrix } \\
\hline & $\mathrm{e}_{2}$ & $e_{3}$ & $e_{4}$ & $e_{5}$ \\
\hline$e_{2}$ & 0 & 0.2 & 0.8 & 0 \\
\hline$e_{3}$ & 0.6 & 0 & 0.4 & 0 \\
\hline$e_{4}$ & 0.4 & 0.6 & 0 & 0 \\
\hline$e_{5}$ & 0.3 & 0.3 & 0.4 & 0 \\
\hline \multicolumn{5}{|c|}{ intersection $v_{6}$ probability matrix } \\
\hline & $e_{6}$ & $e_{7}$ & $e_{8}$ & \\
\hline$e_{6}$ & 0 & 0.2 & 0.8 & \\
\hline$e_{7}$ & 1 & 0 & 0 & \\
\hline$e_{8}$ & 0.4 & 0.6 & 0 & \\
\hline
\end{tabular}

Figure 1: Example of application of the recursive algorithm to estimate the flow of taxis in a road network

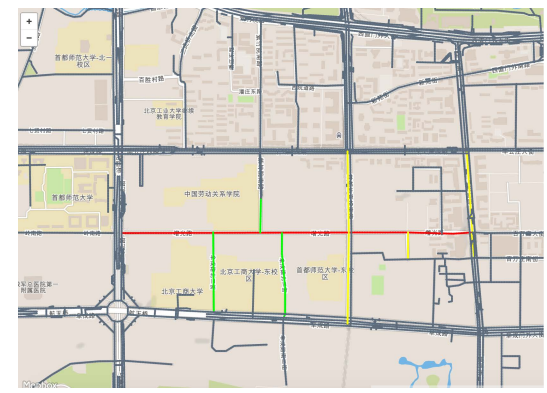

Figure 2: Sample output of web application

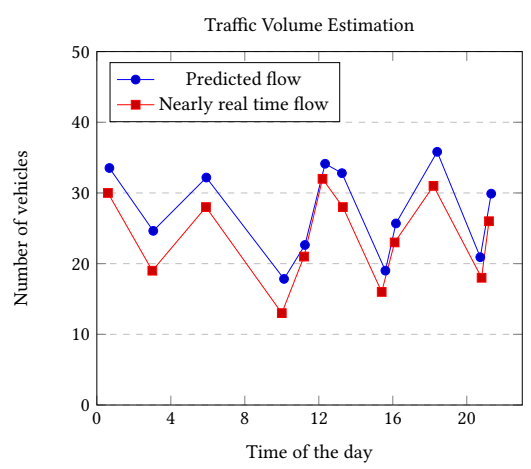

Figure 3: Estimated versus actual values

traffic prediction models. Next steps include the design of load balancing strategies to optimize the allocation of resources to perform computational intensive tasks such as real-time map-matching in parallel with less demanding tasks such as GPS data ingestion or updating counters in near-real time, and the study of data sparsity, which is an important issue when building traffic prediction models, even if very large datasets such as T-Drive are used.

\section{ACKNOWLEDGMENTS}

This work is partially funded by National Funds of the FCT (Foundation for Science and Technology) granted to projects UIDB/00127/2020 and UIDB/04524/2020, and funds of FCT and Fundo Europeu de Desenvolvimento Regional (FEDER), Programa Operacional Competitividade e Internacionalização granted to the project POCI-01-0145FEDER-032636.

\section{REFERENCES}

[1] Ming-Feng Chang, Chi-Hua Chen, Yi-Bing Lin, and Chung-Yung Chia. 2015. The frequency of CFVD speed report for highway traffic. Wireless Communications and Mobile Computing 15, 5 (2015), 879-888.

[2] Taurus T. Dang, Henry Y. T. Ngan, and Wei Liu. 2015. Distance-based k-nearest neighbors outlier detection method in large-scale traffic data. 2015 IEEE International Conference on Digital Signal Processing (DSP) (2015), 507-510.

[3] Merkebe Getachew Demissie, Gonçalo Homem De Almeida Correia, and Carlos Bento. 2013. Intelligent road traffic status detection system through cellular networks handover information: An exploratory study.

[4] Chenjuan Guo, Bin Yang, Jilin Hu, and Christian S. Jensen. 2018. Learning to Route with Sparse Trajectory Sets. In 34th IEEE International Conference on Data Engineering, ICDE 2018. 1073-1084.

[5] Wei Liu, Yu Zheng, Sanjay Chawla, Jing Yuan, and Xing Xie. 2011. Discovering Spatio-Temporal Causal Interactions in Traffic Data Streams. In Proceedings of the 17th SIGKDD conference on Knowledge Discovery and Data Mining (proceedings of the 17th sigkdd conference on knowledge discovery and data mining ed.).

[6] Chuishi Meng, Xiuwen Yi, Lu Su, Jing Gao, and Yu Zheng. 2017. City-wide Traffic Volume Inference with Loop Detector Data and Taxi Trajectories. GIS: Proceedings of the ACM International Symposium on Advances in Geographic Information Systems (2017).

[7] Linsey Xiaolin Pang, Sanjay Chawla, Wei Liu, and Yu Zheng. 2013. On detection of emerging anomalous traffic patterns using GPS data. Data and Knowledge Engineering 87 (2013), 357-373.

[8] Jack Snowdon, Olga Gkountouna, Andreas Züfle, and Dieter Pfoser. 2018. Spatiotemporal traffic volume estimation model based on GPS samples. 5th Intern. ACM SIGMOD Workshop on Managing and Mining Enriched Geo-Spatial Data, GeoRich 2018 (2018), 1-6.

[9] Yilun Wang, Yu Zheng, and Yexiang Xue. 2014. Travel Time Estimation of a Path using Sparse Trajectories. In Proceedings of the 20th SIGKDD conference on Knowledge Discovery and Data Mining (KDD 2014).

[10] Jing Yuan, Yu Zheng, Xing Xie, and Guangzhong Sun. 2011. Driving with knowledge from the physical world. Proceedings of the ACM SIGKDD International Conference on Knowledge Discovery and Data Mining 5 (2011), 316-324.

[11] J. Yuan, Y. Zheng, X. Xie, and G. Sun. 2013. T-Drive: Enhancing Driving Directions with Taxi Drivers' Intelligence. IEEE Transactions on Knowledge and Data Engineering 25, 1 (2013), 220-232.

[12] Jing Yuan, Yu Zheng, Chengyang Zhang, Wenlei Xie, Xing Xie, Guangzhong Sun, and Yan Huang. 2010. T-Drive: Driving Directions Based on Taxi Trajectories. In Proceedings of 18th ACM SIGSPATIAL Conference on Advances in Geographical Information Systems.

[13] Yu Zheng, Licia Capra, Ouri Wolfson, and Hai Yang. 2014. Urban Computing: Concepts, Methodologies, and Applications. ACM Trans. Intell. Syst. Technol. 5, 3, Article 38 (2014), 55 pages.

[14] Yu Zheng, Yanchi Liu, Jing Yuan, and Xing Xie. 2011. Urban Computing with Taxicabs. In Proceedings of the 13th ACM International Conference on Ubiquitous Computing. Ubicomp 2011. 